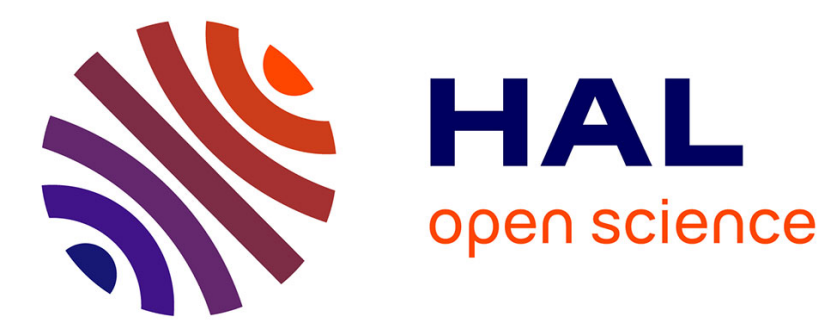

\title{
From measuring electron charge to exploring particle-wave duality: A new didactic experimental approach
}

E. Salançon, Alain Degiovanni, Laurent Lapena, Roger Morin

\section{- To cite this version:}

E. Salançon, Alain Degiovanni, Laurent Lapena, Roger Morin. From measuring electron charge to exploring particle-wave duality: A new didactic experimental approach. American Journal of Physics, 2019, 87 (3), pp.194-199. 10.1119/1.5086392 . hal-02043197

\section{HAL Id: hal-02043197 \\ https://hal-amu.archives-ouvertes.fr/hal-02043197}

Submitted on 27 Feb 2019

HAL is a multi-disciplinary open access archive for the deposit and dissemination of scientific research documents, whether they are published or not. The documents may come from teaching and research institutions in France or abroad, or from public or private research centers.
L'archive ouverte pluridisciplinaire HAL, est destinée au dépôt et à la diffusion de documents scientifiques de niveau recherche, publiés ou non, émanant des établissements d'enseignement et de recherche français ou étrangers, des laboratoires publics ou privés.

\section{(c)(1)}

Distributed under a Creative Commons Attribution| 4.0 International License 


\title{
From measuring electron charge to exploring particle-wave duality: a new didactic experimental approach
}

\author{
Evelyne Salançon,* Alain Degiovanni, Laurent Lapena, and Roger Morin \\ Aix-Marseille Univ., CNRS, CINaM-UMR 7325, Marseille, France
}

( Dated: October 16, 2018)

\begin{abstract}
This paper points out the strong didactic potential of an experiment originally intended for electron source research. Using a dual stage micro-channel plate in a low-energy electron point projection microscope, electrons are spatially detected one by one. Field electron emission affords wide-ranging intensity enabling the electron charge to be determined by comparing counting and analog measurements. The same setup is used to illustrate buildup of an interference pattern from separate low-energy electron detection, thereby enabling the Planck constant to be determined. Both granular and continuous signals are directly observable in this educational approach.
\end{abstract}




\section{INTRODUCTION}

Physics students obtain an understanding of electrons as particles through didactic experiments $^{1-3}$. Examples include measuring the ratio $(q / m)$ between the charge and the mass of the electron to confirm students' physical representation of electrons. When a Millikan experiment ${ }^{4-7}$ or shot noise analysis ${ }^{8,9,16}$ is added, they are also able to measure the electron's charge alone. While these experiments are very useful to confirm the particle behavior of the electron, they are necessarily based on the collective response because there is no individual signal from a single electron.

Electron diffraction experiments ${ }^{10,12-15}$ allow students to approach wave mechanics too. There is even a ready-made experimental setup available from a well-known educational company $^{19}$. In recent years, the particle-wave duality of matter ${ }^{17,18}$, and especially of electrons, was illustrated in different experiments based on the 1963 thought experiment of Richard Feynman ${ }^{20}$. Carried out on a variety of particles (high energy electrons ${ }^{21,22}$, low energy electrons ${ }^{23,24}$, photons ${ }^{25-27}$ ), these Feynman experiments are, however, too complex and costly for educational use.

Here, we use a low-energy electron point projection microscope ${ }^{28-31}$ to independently determine certain particle and wave characteristics of the electron. First, its charge is determined by concurrently using an analog measurement method and a counting measurement method. Its mass can therefore be determined through the deflection in an electric or magnetic field. Then, its particle-wave behavior is observed and measured via the single-electron buildup of an interference pattern. This offers an experimental illustration of the de Broglie law, and the Planck constant can be deduced from these three measurements. In addition, the experiment allows students to directly observe the electron's ability to probe matter.

The granularity of electrons is observable and measurable only if the emission process gives access to several orders of magnitude of electron intensity. Field electron emission meets this condition, offering access to several orders of magnitude of emitted intensity described through the Fowler-Nordheim equation $(1)^{32}$.

$$
I=A(\Phi) \cdot F^{2} \cdot \exp \left(-6.49 \times 10^{9} \frac{\Phi^{\frac{3}{2}}}{F}\right)
$$

with $A(\Phi)$, a pre-factor depending on the work function $\Phi$, and $F$, the electric field applied to the emitter that is given by: $F=\frac{V}{\beta . r}$ for a metal tip of end-radius $r$ supplied to 
the voltage $V$ in front of a mass-connected extractor. $\beta$ is a geometric pre-factor. In the case of a plane emitter/extractor setup, this electric field is simply $F=\frac{V}{d}$, with $d$ the distance between the emitter and the extractor. In practice, a decreasing linear plot $\ln \frac{I}{V^{2}}$ versus $\left(\frac{1}{V}\right)$ is the signature of a field emission process. In this experiment, linearity is observed for 10 orders of magnitude.

\section{EXPERIMENTAL SETUP}

The experiment uses a low-energy electron point projection microscope $\mathrm{e}^{33,34}$ which produces geometric projection shadows and in-line holograms of an object. This object needs to be partially opened in order to obtain a reference beam (Fig. 1). Here, the electron source relies on a field emission process. The source consists of a celadonite insulating crystal placed at the apex of a carbon wire ${ }^{35}$. The intensity is measured using a picoammeter (Keithley 485) floating between the power supply and the source. The object used here is a lacey carbon film (AGS166-3H from AgarScientific): a copper grid covered by a random-holed carbon layer. It is grounded. To increase microscope magnification, the grounded object is moved towards the source (from $2.5 \mathrm{~cm}$ to $100 \mathrm{~nm}$ ) with a piezoelectric actuator (MS30 from Mechonics). This piezoelectric actuator is mounted on the same mechanical system as the source. Rotation of the system makes it possible to turn the beam towards the detector. A dual stage micro-channel plate fluorescent screen assembly (F2225-21P from Hamamatsu) detects single low-energy electrons from 10 to $1000 \mathrm{eV}$. We recently showed that in counting mode, this apparatus enhances the resolution of a low-energy electron microscope ${ }^{36}$. In the present experiment, this mode is used both to determine the electron charge and to show the particle-wave duality. This detector is placed $86 \mathrm{~cm}$ away from the source. Images and films of the fluorescent screen are recorded using a cooled CCD camera (Model C4742-95 from Hamamatsu). Here, a counting mode record is directly obtained from the integrated software HiPic from Hamamatsu: the stain produced by the impact of one electron is reduced to one pixel, and each impact counts as 1.

In this experiment, electron energy is directly determined by the emission voltage $V_{e}$. The emission process being a Fowler-Nordheim type, intensity increases exponentially with voltage. 


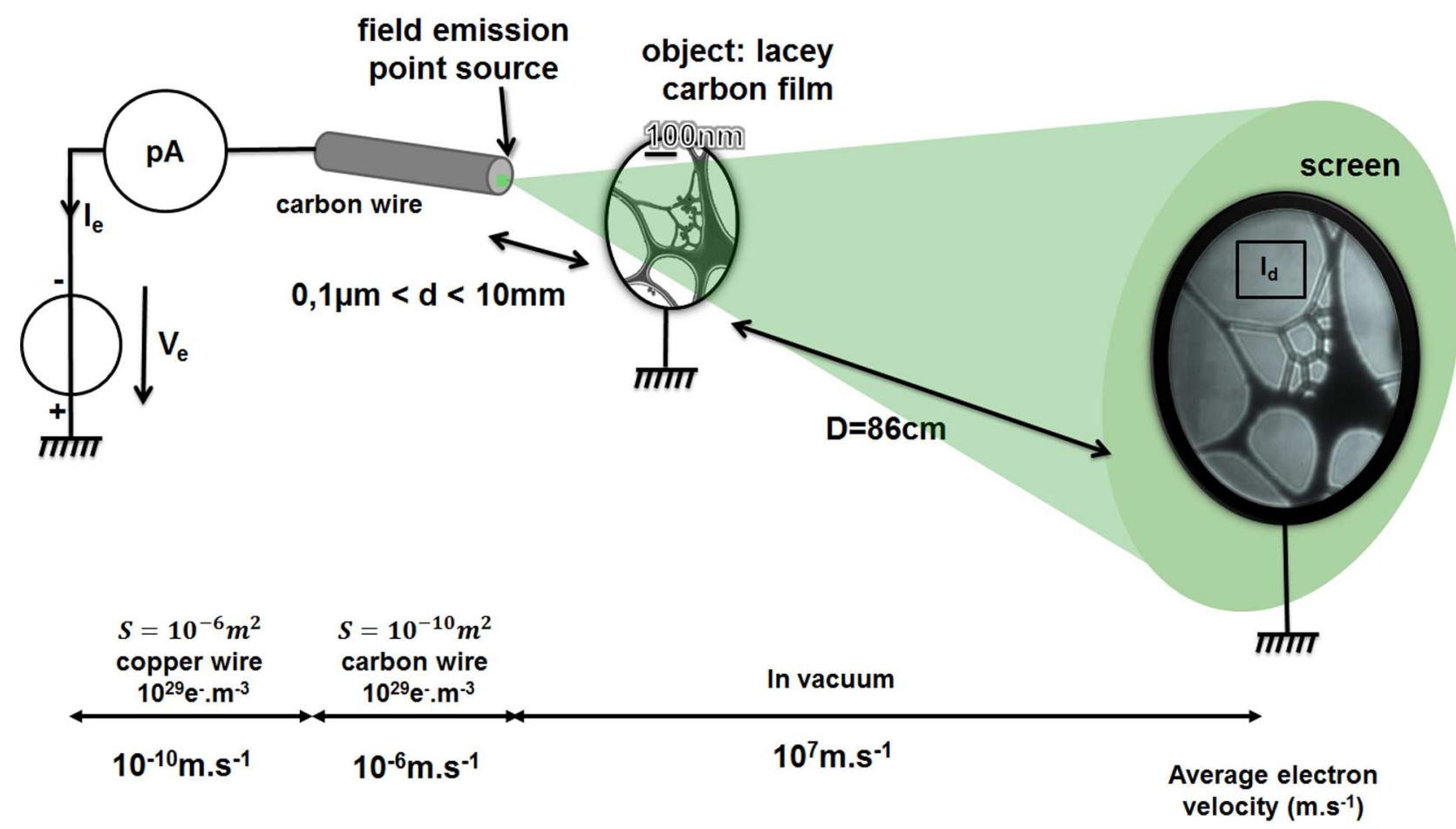

FIG. 1. Low-energy electron microscope. A field emission source, biased to some $V_{e}=-100 \mathrm{~V}$ through a picoammeter, illuminates a lacey carbon film. The physical shadow of the film is observed on a dual stage micro-channel plate fluorescent screen assembly. Example of electron velocities are given for $V_{e}=-1000 \mathrm{~V}$ and $I_{e}=1 \mu \mathrm{A}$.

For teaching purposes, two adjustments to this experiment setup can be envisaged:

- First, the source could be changed, employing a tungsten tip field emitter or any field electron emitter. However, the advantage of using a celadonite source is that the pressure can be 3 orders of magnitude higher, up to about $10^{-6}$ mbar, than with a tungsten tip.

- Second, a cheaper camera could be used and the counting mode treatment performed with a free external plugin like QuickPalm ${ }^{37}$. However, this would mean recording and treating every single event's analog image, which would require significantly increased computer memory, from 225Mo to $3 \mathrm{Go}$. 


\section{ELECTRON CHARGE MEASUREMENT}

To perform the electron charge measurement, the shadow of the lacey carbon film is recorded both in analog and in counting mode (Fig. 2) thanks to the greatly increased electron velocity in the circuit. The velocity of electrons after the grounded object in the experiment is given by the emission voltage as: $v=\sqrt{\frac{2 e V_{e}}{m_{e}}} \sim 10^{7} \mathrm{~m} . \mathrm{s}^{-1}$ with $m_{e}$ the electron mass and $e$, the electron charge. Electrons are detectable one by one as they hit the detector with a high enough energy (or momentum). The electron charge is first determined from measurements at a given emitted current, i.e. at a given voltage $V_{e}$. Then measurements for different $V_{e}$ are plotted via a Fowler-Nordheim plot for statistical analysis leading to more precise determination of the electron charge.

\section{A. Measurements at a given voltage}

The electrical intensity is the emitted charge $q$ in a time $t$ :

$$
I_{e}=\frac{q}{t}=\frac{N_{e} e}{t}
$$

with $N_{e}$ the number of emitted electrons in a time $t$ and $e$ the electron charge.

To enable the electrons to be counted, the measurement has to be performed at very low intensity, as low as $1 \mathrm{pA}$. This is possible with our setup, since only a fraction of the electron beam is detected. To determine the electron charge from Eq. 2, we apply the following procedure:

- First, determine the analog emitted intensity $\left(I_{e}\right)$;

- Second, count the detected hits $\left(n_{d}\right)$;

- Third, determine the ratio of detected hits $\left(n_{d}\right)$ to emitted electrons $\left(N_{e}\right)$, which gives the number of electrons emitted.

\section{Determining analog emitted intensity}

As its name implies, the picoammeter cannot measure intensity below picoamps, which is why the first step is required. We exploit the fact that, in continuous mode, analog intensity 
$I_{e}$ is related to gray level $\overline{g l}(t)$ of the image of an illuminated part of the detector for exposure time $t$.

For example, here (Fig. 2a), the intensity measured for $V_{e}=-440 \mathrm{~V}$ corresponds to $I_{e}=I_{440}=10 \mathrm{pA}$, the average gray level measured on the illuminated part on Fig.2a is $\overline{g l_{440}}=2030$ for an image composed of 10 frames of $10 \mathrm{~s}\left(t_{440}=100 \mathrm{~s}\right)$ and a channel plate applied voltage of about $1.2 \mathrm{kV}$. Thus, the analog calibration of the gray level for this channel plate amplification is:

$$
I_{e}=\frac{\overline{g l}}{t} \times \frac{t_{440}}{\overline{g l_{440}}} \times I_{440}
$$

The following factors require particular attention:

- image noise: each image needs to be corrected with a long exposure time noise image,

- image saturation: amplification of the channel plate and frame time need to be tuned to prevent saturation,

- channel plate amplification: if the amplification is changed, recalibration of Eq. 3 is required, using measurements made before and after the change,

- area used to find the $\overline{g l}$ on each image (size of rectangles in Fig. 2), which has to be virtually identical between any two measurements.

\section{Counting detected hits}

For low intensity $10^{-3}<I_{e}<10^{-1} \mathrm{pA}$ an image can be recorded in analog mode and in counting mode in the highest-intensity region detected. For $V_{e}=-350 \mathrm{~V}$, Fig. 2b gives an analog intensity deduced from gray level analysis of about $I_{350 \mathrm{~V}}=(3.51 \pm 0.05) \times 10^{-3} \mathrm{pA}$. Fig. 2c, the counting mode image for an exposure time $t=555 \mathrm{sec}$, gives an average number of hits $n_{d}=19.4$ hits/pxl. Because the number of hits in the shadow of the grid should be null, the signal-to-noise ratio $(S / N)$ is measured directly on the image. $(S / N)$ is the ratio between the gray level in the illuminated part and in the shadow of the grid. In Fig.2c $(S / N)=500$ and in Fig. 2d $(S / N)=10$. Fig. 2d shows the lowest signal-tonoise ratio measured in this work, with the uncertainty on the number of detected hits $n_{d}=(0.060 \pm 0.006) \mathrm{hits} / \mathrm{pxl}$. 


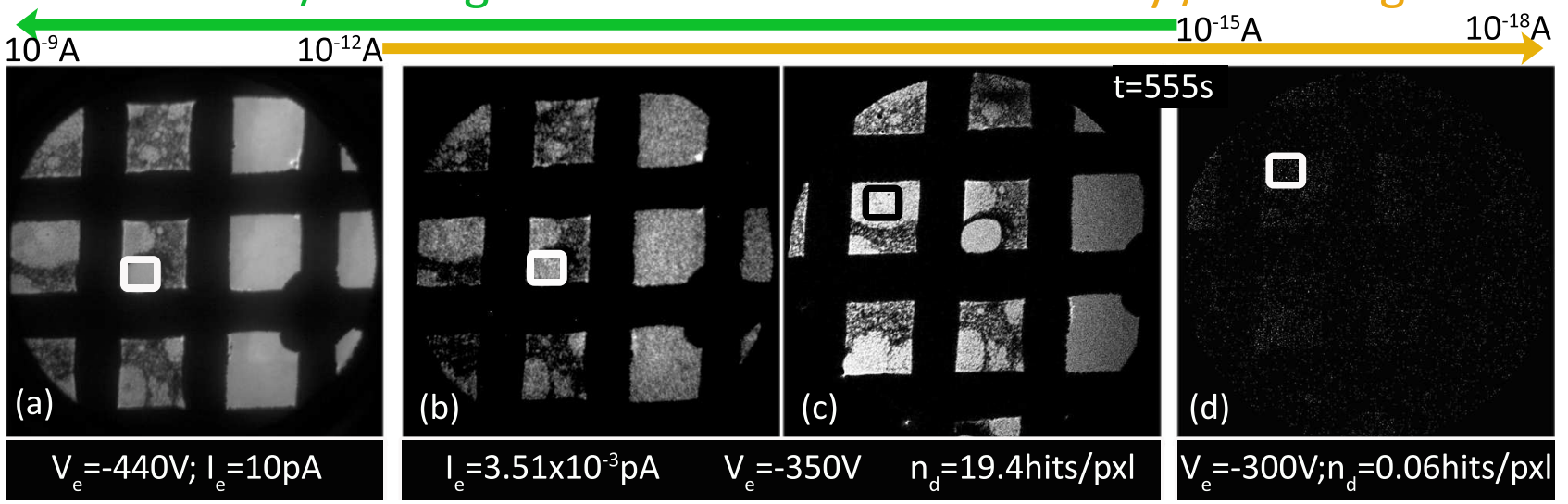

FIG. 2. Depending on electron detection, continuous intensity or granularity of electrons are illustrated (a) For a few picoamps, picoammeter and gray level detection are required; gray level gives the intensity calibration; (b) For a few femtoamps, analog intensity is measured through the gray level when (c) the counting mode gives the number of detected hits; (d) For a few attoamps, the number of detected hits is given for the highest-intensity zone. Measurements are performed in rectangles.

\section{Determining ratio of detected hits to emitted electrons}

To determine this ratio, we need to know the angular distribution of emission and the electron detection efficiency of the detector.

The electron's angular distribution in the cone angle is measured by rotating the source/sample assembly with respect to the detector. It is here assumed that the solid angle is the same whatever the emission voltage. Fig. 3 shows 3 meshes of the copper grid (900 pixels). The shadow actually extends along 12 meshes, but the detector is not large enough to detect the entire angular distribution. On the accessible signal, intensity is almost normally distributed (plot in in Fig.3b) with a standard deviation $\sigma=(300 \pm 50)$ pxls, determined by fitting the experimental curve. Assuming that the intensity distribution is isotropic, the emission area in the plane of the detector is obtained by integrating the normal distribution determined over the area, $S=2 \pi \sigma^{2}$. However, this yields major uncertainty:

$$
S=(6 \pm 2) \times 10^{5} \text { pxls }
$$


The manufacturer of the channel-plate specifies $60 \%$ for the open area ratio, with a detection efficiency $\eta$ that varies from 50 to $85 \%$ depending on the electron energy, open area ratio and electron angle incidence ${ }^{38}$. This means that the actual number of electrons is about $(1.4 \pm 0.4)$ times the number of hits. Therefore, the number of emitted electrons is given by:

$$
N_{e}=1.4 \times S \times n_{d}
$$

with $n_{d}$ the number of detected hits per pixel measured at the highest signal intensity and $S$ the emission area in pixels.
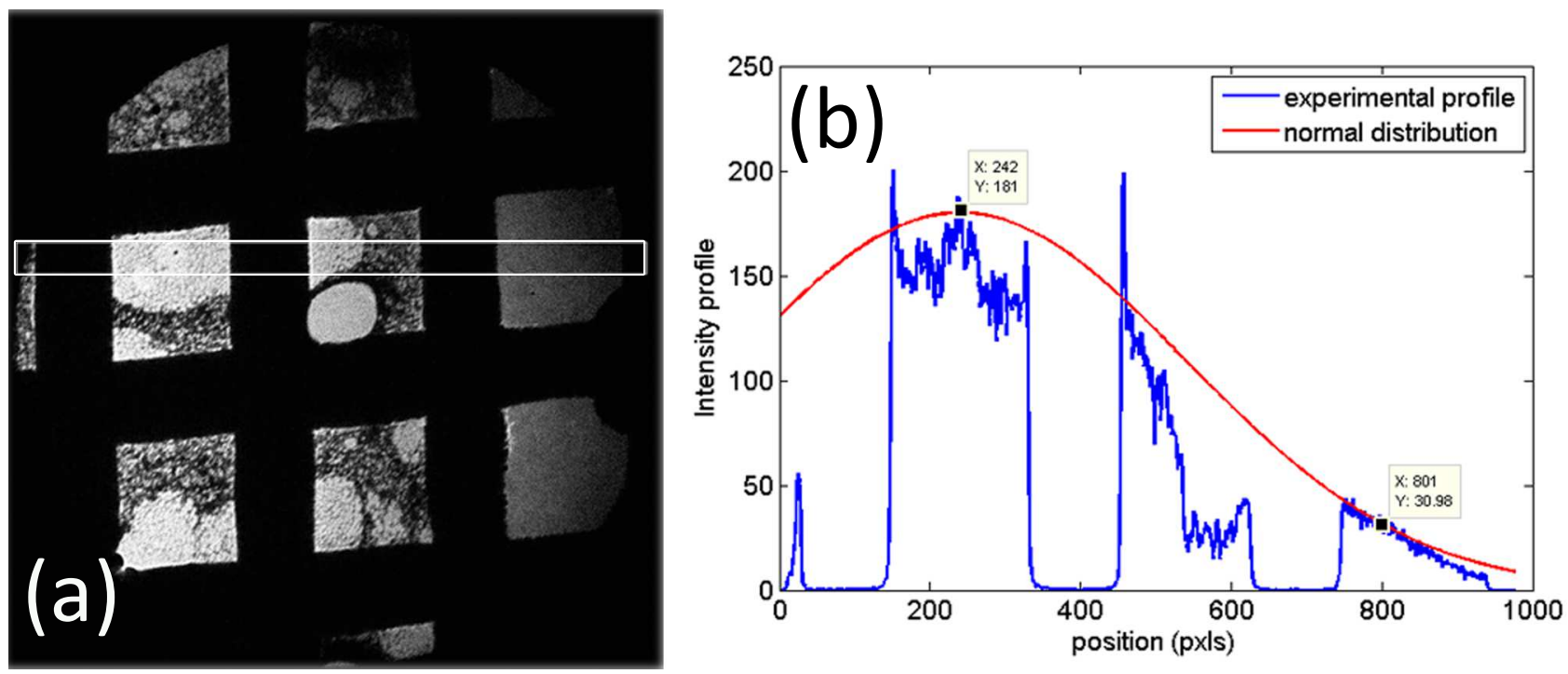

FIG. 3. Measurement of the intensity distribution in the cone angle (a) counting mode imaging of the sample shadow $(V=-360 \mathrm{~V}, I=0.012 \mathrm{pA})$; (b) in blue: intensity profile along the white rectangle in (a) and in red: normal distribution with a standard deviation $\sigma=(300 \pm 50)$ pxls.

From this direct analysis of the data obtained in the above three steps, the electron charge was here determined at $V_{e}=-350 \mathrm{~V}$ :

$$
e=\frac{I_{e}}{\frac{1.4 \times S \times n_{d}}{t}}=\frac{3.51 \times 10^{-3} \times 10^{-12}}{\frac{1.4 \times 6 \times 10^{5} \times 19.4}{555}}=1.2 \times 10^{-19} \mathrm{C}
$$

Given the accumulation of uncertainties here, this result is highly satisfactory:

- the pre-factor in analog mode is determined for each amplification change;

- the distribution in the detector plane is approximated to a normal law and is not measured everywhere (which would be possible with a more precise angular distribution 
measurement);

- the number of detected hits depends on the threshold used. For example, with Fig.2d at $V_{e}=-300 \mathrm{~V}$, the highest-intensity region is still visible but the signal-to-noise ratio is clearly lower than in other cases $((S / N)=10)$. If the threshold used for the counting mode is too low, false hits are detected; if it is too high, hits are lost. For the highest-intensity measurements, the threshold is fixed with reference to the gray level distribution without a signal: the threshold is fixed at the upper limit of this measurement, giving a signal-to-noise ratio of up to 4000. For the lowest-intensity measurements, the threshold is fixed so as to obtain a signal-to-noise ratio of about 10.

We then used this method to perform electron charge measurement for different power supplies. The values in Tab.I clearly illustrate the accumulated errors: for the highest intensity levels measured (about $0.1 \mathrm{pA}$ ), the number of detected hits seems overestimated and for the lowest intensity levels (under $0.0001 \mathrm{pA}$ ), the number of hits is underestimated. This is due in one case to false hits and in the other case to the poor signal-to-noise ratio. To obtain a more representative value, measurements were performed subsequently over a large voltage range.

TABLE I. Measurements for $t=555 \mathrm{sec}$ and corresponding calculated electron charge for 3 power supply conditions

\begin{tabular}{lccl}
\hline \hline$V_{e}(\mathrm{~V})$ & -330 & -350 & -400 \\
\hline$I_{e}(\mathrm{pA})$ & $4.7 \times 10^{-4}$ & $3.51 \times 10^{-3}$ & $3.8147 \times 10^{-1}$ \\
$n_{d}(\mathrm{hits} / \mathrm{pxl})$ & 1.4 & 19.4 & 3336.4 \\
$e(\mathrm{C})$ & $2.2 \times 10^{-19}$ & $1.2 \times 10^{-19}$ & $0.8 \times 10^{-19}$ \\
\hline \hline
\end{tabular}

\section{B. Measurements over a large voltage range}

The objective here was to provide more robust results by decreasing uncertainty on analog and counting measurements. These measurements are not defined by their actual value but rather by their value on the Fowler-Nordheim fit obtained in each mode. A statistical study 
was performed over a large intensity range. Fig.4a is the $I(1 / V)$ semi-log plot in analog mode and $\left(N_{e} / t\right)(1 / V)$ in counting mode with $\frac{N_{e}}{t}=\frac{1.4 \times S \times n_{d}}{t}$. Right $\left(N_{e} / t\right)$ and left $\left(I_{e}\right)$ scales have the same magnitude and we can pass from one to the other by multiplying the right scale by the charge of the electron. To determine this charge in Fig.4b, the right scale is shifted so that the data fits from analog and counting modes are perfectly aligned. Here, the different experimental data fitted with the same slope, $V_{0}=-5461 \mathrm{~V}$, and gave an $\mathrm{R}$ squared of about $98 \%$. Note that the actual measurements at $-330 \mathrm{~V},-350 \mathrm{~V},-400 \mathrm{~V}$ in analog and in counting modes are not exactly on their respective fits. The emitted intensity is now directly comparable to the electron emission rate (i.e. $10^{-6} \mathrm{pA}=e \times 8 \mathrm{e}^{-} / \mathrm{s}$ ). From this procedure, the electron charge is found to be $e=1.3 \times 10^{-19} \mathrm{C}$.

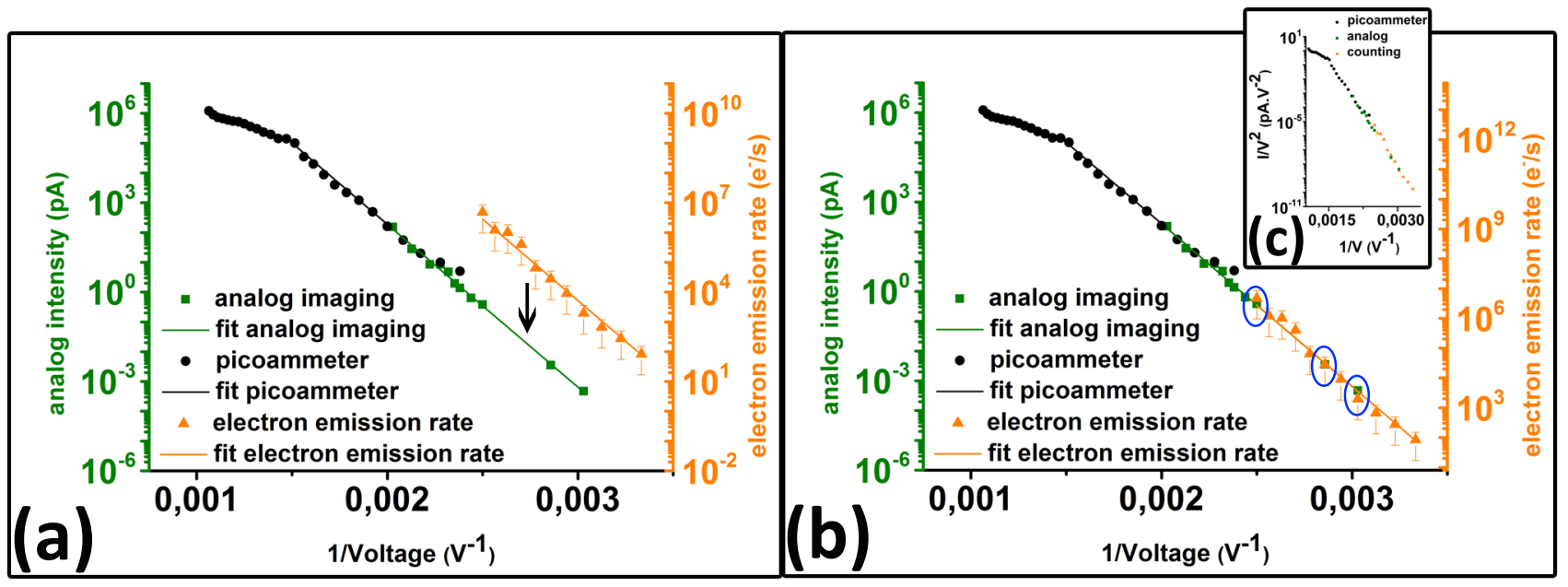

FIG. 4. (a) Black and green dots: Intensity versus inverse voltage for analog measurements (picoammeter and gray level measurements); Orange: electron emission rate deduced from counting mode imaging; (b) Alignment of data fits from picoammeter, analog mode and counting mode measurements; these fits are exponential decays with a constant of $V_{0}=-5461 \mathrm{~V}$; blue circles: single measurements at $V_{e}=-330 \mathrm{~V},-350 \mathrm{~V}$ and-400 V; (c) Fowler-Nordheim plot observable below 10 orders of magnitude using the full dataset.

Clearly, here, the error that most impacts electron charge measurements is the error on the angular distribution of electron emission and on dual micro-channel plate efficiency. Given the uncertainties on these values, the calculated uncertainty on the determined electron charge is particularly large. Thus, the electron charge found here is:

$$
e=(1.3 \pm 0.8) \times 10^{-19} \mathrm{C}
$$


To approach the actual SI base units $e=1.602 \times 10^{-19} \mathrm{C}$, channel-plate efficiency should be clarified and angular distribution should be measured more precisely. One way of doing this would be to place the detector closer to the source, enabling the whole signal to be measured.

Note that the Fowler-Nordheim curve for these intensity measurements plotted in Fig.4c confirms the process involved here as field electron emission. For the highest intensities $\left(I_{e}>1 \mu \mathrm{A}\right)$, the plot changes and shows a level of saturation previously observed for this kind of source ${ }^{35}$. Field emission is observed for more than 10 orders of magnitude.

\section{DETERMINING PLANCK CONSTANT}

The electron charge was measured with the object far away from the source and a minimum emission voltage of $-300 \mathrm{~V}$ to obtain a reasonable signal-to-noise ratio. At this voltage, the average electron emission rate is 100 electrons per second. The velocity of electrons after the grounded object is about $v=10^{7} \mathrm{~m} \cdot \mathrm{s}^{-1}$. The experimental apparatus being almost one meter long, electrons are in the experiment for about $0.1 \mu \mathrm{s}$. Although the source is not yet known to be a single electron source, it is possible to perform a kind of Feynman thought experiment involving particle-by-particle double slit interference pattern recording.

To perform such a double slit experiment, two "virtual sources" can be obtained by using an electrostatic biprism ${ }^{11}$. In the lacey carbon film, some carbon filaments are very small and a cylindrical attractive field often appears surrounding them. This results either from the electrostatic influence of the source when the hole around the filament is large compared to the source-to-filament distance, or from positive charging under the electron beam when

the secondary electron coefficient is larger than 1 . This field deflects the electron beam which, after crossing the object, seems to come from two virtual sources placed in the real source plane ${ }^{29}$ (Fig.5a).

For example, the interference pattern shown in Fig.6 was obtained for a source-object distance $d=(19 \pm 1) \mu \mathrm{m}$ and the filament observed had a diameter about $\phi=(118 \pm 9) \mathrm{nm}$ (see Fig.5c: the scanning electron micrograph of the lacey carbon film). The experiment is carried out at $V_{e}=-227 \mathrm{~V}$. The electrical intensity is about $10 \mathrm{pA}$, corresponding to an average distance $l=14 \mathrm{~cm}$ between 2 electrons. Images are recorded every $t_{f}=10 \times 111 \mathrm{~ms}$. At this rate, an average number of 100 electrons are detected per frame on the whole 


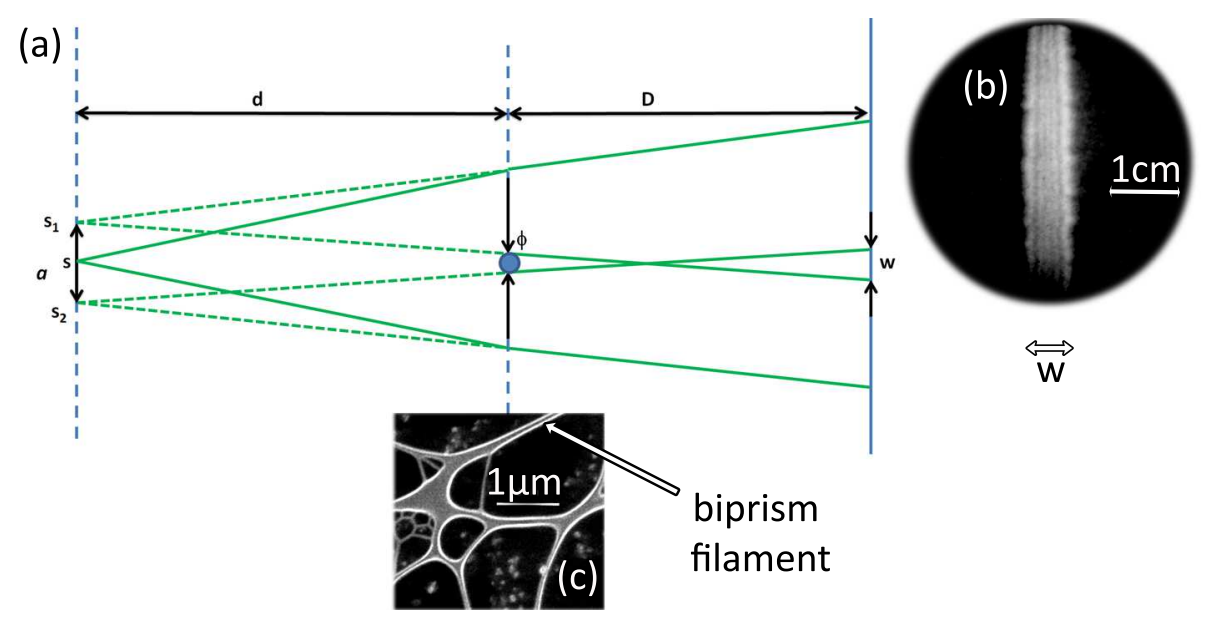

FIG. 5. (a) Geometrical representation of the experiment where $s$ is the real source and $\phi$, the biprism diameter; $s_{1}$ and $s_{2}$ are the "virtual sources" (b) Analog recording of the electron projection of the biprism: interference pattern from $s_{1}$ and $s_{2}$ in the plane of the detector appears in $w$; (c) Scanning electron microscope view of the filament used as biprism in the experiment, width about $\phi=118 \mathrm{~nm}$.

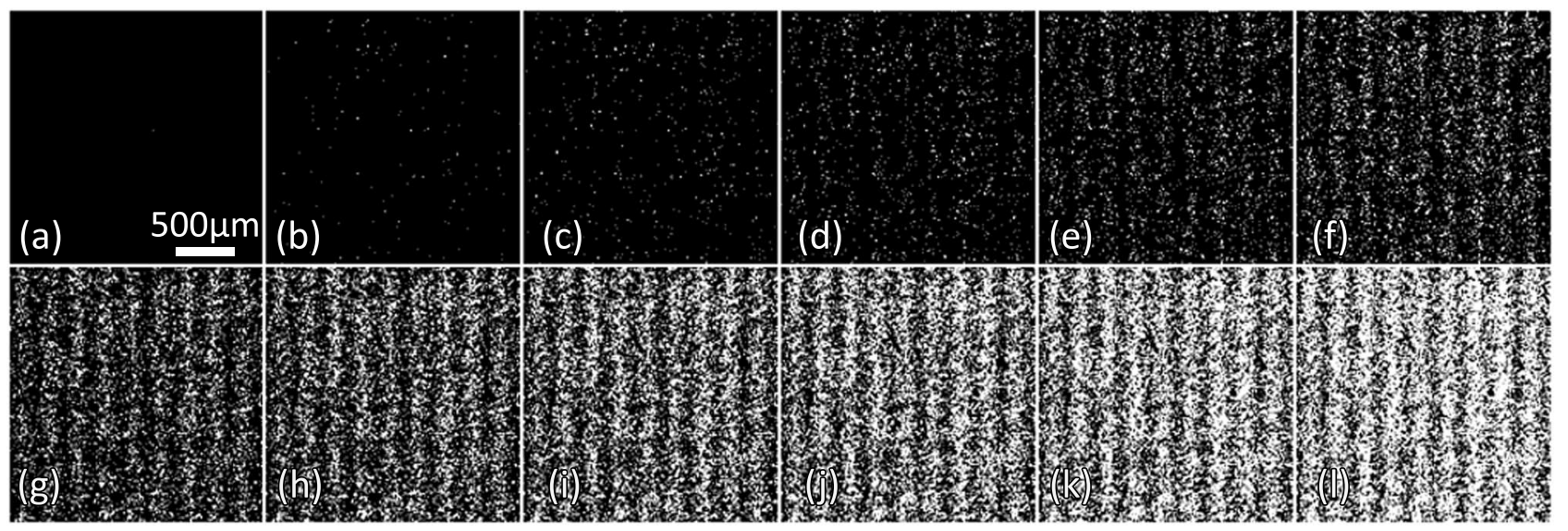

FIG. 6. Interference pattern buildup. Emission voltage $V_{e}=-227 \mathrm{~V}$, source-object distance $d=18 \mu \mathrm{m}$, inter-source distance $a=294 \mathrm{~nm}$, object-detector distance $D=86 \mathrm{~cm}$. The fringe distance $i=0.24 \mathrm{~mm}$. (a) $t=10 \times 111 \mathrm{~ms}$; (b) $t=11 \times 10 \times 111 \mathrm{~ms} ;($ c) $t=21 \times 10 \times 111 \mathrm{~ms}$;
(d) $\quad t=31 \times 10 \times 111 \mathrm{~ms}$;
(e) $\quad t=41 \times 10 \times 111 \mathrm{~ms}$;
(f) $t=51 \times 10 \times 111 \mathrm{~ms}$;
$t=61 \times 10 \times 111 \mathrm{~ms} ;$
(h) $\quad t=71 \times 10 \times 111 \mathrm{~ms}$;
(i) $\quad t=81 \times 10 \times 111 \mathrm{~ms}$;
$t=91 \times 10 \times 111 \mathrm{~ms} ;(\mathrm{k}) t=102 \times 10 \times 111 \mathrm{~ms}$; $(\mathrm{l}) t=112 \times 10 \times 111 \mathrm{~ms}$.

detector. Fig. 6 corresponds to $(1.5 \times 1.5) \mathrm{mm}^{2}$ on the detector; the interference pattern appears clearly after one minute (see Fig.6g). 
The measured distance between fringes is $i=\frac{\lambda D}{a}=0.24 \mathrm{~mm}$. The inter-source distance $a$, found by geometrical analysis from Fig.5a, is: $a=\frac{w d+\phi(d+D)}{D}$. Fig.5b shows a full view of the detector and gives: $w=(0.72 \pm 0.05) \mathrm{mm}$, then $a=(280 \pm 30) \mathrm{nm}$, the object-detector distance $D=86 \mathrm{~cm}$, giving a wavelength $\lambda=(0.08 \pm 0.01) \mathrm{nm}$.

By using the deBroglie relationship, $\lambda=\frac{h}{\sqrt{2 m_{e} E}}$, the Planck constant is deduced from the wavelength measurement $\lambda=(0.08 \pm 0.01) \mathrm{nm}$, the electron mass $m_{e}=9.31 \times 10^{-31} \mathrm{~kg}$ and the electron energy $E=e V_{e}=227 \mathrm{eV}$ :

$$
h=(6.5 \pm 0.8) \times 10^{-34} \mathrm{~kg} \cdot \mathrm{m}^{2} \cdot \mathrm{s}^{-1}
$$

\section{CONCLUSION}

This paper describes an experiment originally intended for electron source research but which has strong didactic potential. Using a low-energy electron projection microscope, students can observe electron signals in two modes: continuous signals resembling a closed circuit and granular signals indicating individual electrons. This second mode enables an interference pattern buildup, which allows scope for Feynman thought experiments and measurement of the Planck constant. A physical law, the Fowler-Nordheim law describing the emission of electrons by a field effect, makes it possible via statistical treatment to enhance the precision of charge measurement. The advantage of using a projection microscope is that important notions like source, object, imaging, magnification become easily accessible to any student using a simple light point source. Such experiments also provide opportunities to raise more subtle questions, like the role of the detection process in image formation, the trajectory of a particle or the diffraction of a wave. This experiment is used in highlevel university courses like masters' degrees in physics, as well as in vocational training for secondary school teachers. A teachers' group is already using experimental data from this training to prepare movies for young students in France, where particle-wave duality concepts are on the secondary school curriculum.

\section{ACKNOWLEDGMENTS}

The authors would like to thank Marjorie Sweetko for improving the English of this article and IRES (institute of research in science education) teachers' group for its filming work 
(Florence Delorme, Josiane Lliteras, Cédric Michel, Sofia Kellaf and Renaud Boudant).

* salancon@cinam.univ-mrs.fr; permanent address: Campus de Luminy-case 913, 13288 Marseille cedex 9

1 Paul J. Angiolillo, On thermionic emission and the use of vacuum tubes in the advanced physics laboratory, Am. J. Phys. 77 (12), 1102-1106 (2009).

2 William J. Thompson, Determining e/m with a Bainbridge tube: Less data, more physics, Am. J. Phys. 58 (10), 1019-1020 (1990).

3 Joseph E. Price, Electron trajectory in an e/m experiment, Am. J. Phys. 55 (1), 18-22 (1987).

4 Christoph Müller-Hill, Peter Heering, Control and stabilization: making Millikans oil drop experiment work, Eur. J. Phys. 32 (5), 1285-1291 (2011).

5 Ray C. Jones, The Millikan oildrop experiment: Making it worthwhile, Am. J. Phys. 63 (11), 970-978 (1995).

6 Mark A. Heald, Millikan Oil-Drop Experiment in the Introductory Laboratory, Am. J. Phys. 42 (3), 244-246 (1974).

7 J.I. Kapusta, Best measuring time for a Millikan oil drop experiment, Am. J. Phys. 43 (9), 799-801 (1975).

8 Yaakov Kraftmakher, A shot-noise experiment with computer control and data acquisition, Am. J. Phys. 73 (10), 984-986 (2005).

9 James A. Earl, Undergraduate Experiment on Thermal and Shot Noise, Am. J. Phys. 34 (7), $575-580(1966)$.

10 C. Jnsson, Electron Diffraction at Multiple Slits, Am. J. Phys. 42, 4-12 (1974).

11 O. Donati, G.F. Missiroli and G. Pozzi,An Experiment on Electron Interference, Am. J. Phys. 41 (5), 639-644 (1973).

12 P.G. Merli, G.F. Missiroli and G. Pozzi, On the statistical aspect of electron interference phenomena, Am. J. Phys. 44 (3), 306-308 (1976).

13 G. Matteucci and G. Pozzi, Two further experiments on electron interference, Am. J. Phys. 46 (6), 619-624 (1978).

14 S. Frabboni, G.C. Gazzadi and G. Pozzi, Youngs double-slit interference experiment with electrons, Am. J. Phys. 75 (11), 1053-1056 (2007). 
15 S. Frabboni, C. Frigeri, G.C. Gazzadi and G. Pozzi, Two and three slit electron interference and diffraction experiments, Am. J. Phys. 79 (6), 615-619 (2011).

16 D.R. Spiegel, R.J. Helmer, Shotnoise measurements of the electron charge: An undergraduate experiment, Am. J. Phys. 63 (6), 554-560 (1995).

17 O. Nairz, B. Brezger, M. Arndt, A. Zeilinger, Diffraction of complex molecules by structures made of light, PRL 87 (16), 160401-160405 (2001).

18 O. Carnal, J. Mlynek, Youngs double-slit experiment with atoms: A simple atom interferometer, PRL 66 (21), 2689-2693 (1991).

19 'Electron Diffraction', Phywe https://www.phywe.com/en/electron-diffraction.html.

20 Richard P. Feynman, Robert B. Leighton, and Matthew Sands, The Feynman Lectures on Physics, Vol. 1 (Addison-Wesley, 1964).

21 A. Tonomura, J. Endo, T. Matsuda, T. Kawasaki, H. Ezawa, Demonstration of singleelectron buildup of an interference pattern, Am. J. Phys. 57 (2), 117-121 (1989).

22 S. Frabboni, G.C. Gazzadi, V. Grillo, G. Pozzi, Elastic and inelastic electrons in the double-slit experiment: A variant of Feynmans which-way set-up, Ultramicroscopy 154 (21), 49-56 (2015).

23 J.Y. Chesnel, A. Hajaji, R.O. Barrachina, F. Frémont, Young-type experiment using a singleelectron source and an independent atomic-size two-center interferometer, PRL 98 (4), 100403 (2007).

24 R. Bach, D. Pope, S.H. Liou, H. Batelaan, Controlled double-slit electron diffraction, New J. of Physics 15, 033018 (2013).

25 V. Jacques, E. Wu, T. Toury, F. Treussart, A. Aspect, P. Grangier, J.F. Roch, Single-photon wavefront-splitting interference, Eur. Phys. J. D 35 (3), 561-565 (2005).

26 T.L. Dimitrova and A. Weis, The wave-particle duality of light: A demonstration experiment, Am. J. Phys. 76, 137-143 (2008).

27 R.S. Aspden, M.J. Padgett and G.C. Spalding, Video recording true single-photon double-slit interference, Am. J. Phys. 84 (9), 671-678 (2016).

28 W. Stocker, H.W. Fink and R. Morin, Low-energy electron and ion projection microscopy, Ultramicroscopy 31, 379-384 (1989).

29 J. Bardon, A. Degiovanni, V. Georges, and R. Morin, Conducting, semiconducting and insulating objects observed by low-energy electron holography, Ultramicroscopy 92, 133-142 (2002). 
30 A. Eisele, B. Völkel, M. Grunze, A. Gölzhäuser, Nanometer resolution holography with the low energy electron point source microscope, Zeitschrift fr Physikalische Chemie 222 (5-6), 779-787 (2008).

31 J.N. Longchamp, S. Rauschenbach, S. Abb, C. Escher, T. Latychevskaia, K. Kern, H.W. Fink, Proceedings of the nat. academy. of Sciences of USA 114 (7), 1474 (2017).

32 R.G. Forbes, Physics of generalized Fowler-Nordheim-type equations, J. Vac. Sci. Technol. B 26 (2), 788-793 (2008).

33 H.W. Fink, W. Stocker, H. Schmid, Holography with low-energy electrons, PRL 65, 1204-1206 (1990).

34 R. Daineche, A. Degiovanni, O. Grauby, R. Morin, Source of low-energy coherent electron beams, Appl. Phys. Lett 88, 023101 (2006).

35 E. Salançon, R. Daineche, O. Grauby, R. Morin, Single mineral particle makes an electron point source, J. Vac. Sci. Technol. B 33, 0306601 (2015).

36 E. Salançon, A. Degiovani, L. Lapena, R. Morin, High spatial resolution detection of low-energy electrons using an event-counting method, application to point projection microscopy, RSI 89, 043301 (2018).

37 R. Henriques, M. Lelek, E.F. Fornasiero, F. Valtorta, C. Zimmer, M.M. Mhlanga, QuickPALM: 3D real-time photoactivation nanoscopy image processing in ImageJ, Nature Method 7, 339-340 (2010).

38 M. Galanti, R. Gott, J.F. Renaud, A High Resolution, High Sensitivity Channel Plate Image Intensifier for Use in Particle Spectrographs, RSI 42, 1818-1823 (1971). 